\title{
Noise pollution in hospitals
}

\author{
Excessive noise is damaging for both patients and staff
}

\author{
Andreas Xyrichis senior lecturer ${ }^{1}$, John Wynne reader in sound arts $^{2}$, Jamie Mackrill freelance \\ researcher ${ }^{3}$, Anne Marie Rafferty professor of nursing policy ${ }^{1}$, Angus Carlyle professor of sound \\ and landscape ${ }^{2}$
}

${ }^{1}$ Florence Nightingale Faculty of Nursing, Midwifery, and Palliative Care, King's College London, UK; ${ }^{2}$ London College of Communication, University of the Arts London, UK; ${ }^{3}$ Warwick, UK

Noise in hospitals is a common grievance among patients, families, and staff. ${ }^{1}$ In the US, "quietness of hospital environment" is among the lowest scoring items on patient surveys. ${ }^{2}$ In the UK, $40 \%$ of hospital patients are bothered by noise at night, a consistent finding of the NHS Inpatient Survey. ${ }^{1}$ Hospital noise is a steadily worsening problem, with levels regularly exceeding international recommendations. ${ }^{34}$ Noise levels over $100 \mathrm{~dB}$ have been measured in intensive care units, ${ }^{4}$ the equivalent of loud music through headphones and the point beyond which damage to hair cells in the ear can occur.

\section{Effects on patients and staff}

Excessive noise in hospitals reduces the intelligibility of speech and impairs communication, causing annoyance, irritation, and fatigue $^{5}$ and reducing the quality and safety of healthcare. It has been implicated in the development of intensive care psychosis, hospitalisation induced stress, increased pain sensitivity, high blood pressure, and poor mental health..$^{5-7}$

Hospital noise disrupts sleep; machine sounds in particular have a greater negative effect on arousal than human voices. ${ }^{8}$

Post-hospitalisation recovery is also compromised. In one study from Sweden, coronary care patients treated during noisy periods had a significantly higher incidence of rehospitalisation than those treated during quieter periods. ${ }^{9}$ For hospital staff, high noise levels can impact negatively on communication, performance, wellbeing, and caring behaviour, and can contribute to burnout. ${ }^{56}$

Patients report being ill equipped and ill prepared to deal with hospital noise. ${ }^{10}$ Noise can have a cumulative effect: when hospitalised for several nights, patients can feel trapped in a stress inducing soundscape, leading to requests for premature discharge and heightened risk of poor recovery and readmission.

\section{Slow progress}

Three key challenges hinder progress in this area. Firstly, noise is often incorrectly equated with high sound pressure levels. In physics, noise and sound are conceptually indistinguishable. With encultured perception, however, noise is defined as unwanted, uncontrollable, or unpredictable sound. Dripping taps may register low sound pressure levels yet still be considered noisy. A narrow focus on sound pressure levels will not ensure a reduction in noise perception.

Secondly, noise is difficult to measure reliably. Materials used for hospital floors and walls, ward layout, acoustic properties of furnishings, and variables including bed occupancy all affect how sound is perceived. Published studies of noise measurements on wards usually report snapshot recordings over a 24 hour period, and rarely give detailed contextualisation of those readings. Validated measurement instruments are also lacking. One preliminary but promising approach uses a two dimensional plot of patients' subjective responses to hospital sounds as a visual tool to evaluate noise reduction interventions. $^{11}$

Thirdly, there are many potential sources of noise in hospitals. Alarms, televisions, trolleys, and phones, as well as staff, visitor, and patient conversations are reported as common sources of disturbance. ${ }^{12}$ Not all loud sounds are perceived as noise by patients, however. Some find the sound of the tea trolley pleasing, ${ }^{13}$ and some intensive care unit patients welcome ringing telephones as a sign that they are not alone. ${ }^{14}$

Interventions to date have included earplugs, noise warning systems, sound absorbing panels, educational initiatives, and noise reduction protocols. ${ }^{15-18}$ Evidence suggests possible benefits, but the lack of randomisation, blinding, control groups, and long term follow up, and the multi-component nature of many interventions make it difficult to isolate the effectiveness of a single initiative. Moreover, patients and relatives are rarely involved in this research; they are seen as passive recipients of the hospital soundscape rather than active participants in its creation. In the absence of firm evidence, future solutions should be based on a careful assessment of each hospital environment, and designed with input from all stakeholders, particularly patients and families. 
Patients and families need clear information about likely noise levels during admissions so they are better prepared and can consider simple solutions such as headphones with their own choice of audio content. User friendly guides on the potential sources of noise on wards can also help. Education for staff is also needed, to encourage a culture that considers noise reduction an integral part of safe high quality healthcare.

Finally, early investigations of sound masking and noise cancellation technology suggest potential for use in healthcare. ${ }^{19}$ Sound masking - the addition of background, broadband sound (such as white noise) which can be optimised for particular environments to reduce noise induced disturbance-has been shown in a non-randomised trial to significantly improve sleep in hospitals, for example. ${ }^{20}$

For too long, noise in hospitals has been an intractable problem. Researchers must expand their focus from quantitative reductions in sound pressure levels to broader qualitative improvements in hospital soundscapes. Full patient partnership in this research will help accelerate progress in what has been an unacceptably slow moving field.

\section{Not commissioned, peer reviewed}

Competing interests: We have read and understood BMJ policy on declaration of interests and declare that $\mathrm{AX}, \mathrm{JW}, \mathrm{JM}, \mathrm{AC}$ and $\mathrm{AMR}$ received funding from the Cultural Institute at King's as part of the HPNoSS project, which used equipment donated by Bose, Sound Directions, and Acoustic Sheep.

Raleigh V, Thompson J, Jabbal J, et al. Patients' experience of using hospital services. Picker Institute Europe, 2015.

2 Hospital Consumer Assessment of Healthcare Providers and Systems. www.hcahpsonline. org.

3 Busch-Vishniac IJ, West JE, Barnhill C, Hunter T, Orellana D, Chivukula R. Noise levels in Johns Hopkins Hospital. J Acoust Soc Am 2005;118:3629-45. 10.1121/1.2118327 16419808
4 Darbyshire JL. Excessive noise in intensive care units. BMJ 2016;353:i1956. 10.1136/bmj.i1956 27059670

5 Basner M, Babisch W, Davis A, et al. Auditory and non-auditory effects of noise on health Lancet 2014;383:1325-32. 10.1016/S0140-6736(13)61613-X 24183105

6 Grumet GW. Pandemonium in the modern hospital. N Engl J Med 1993;328:433-7. 10.1056/NEJM199302113280612 8421463

7 Choiniere DB. The effects of hospital noise. Nurs Adm Q 2010;34:327-33. 10.1097/NAQ.0b013e3181f563db 20838178

8 Buxton OM, Ellenbogen JM, Wang W, et al. Sleep disruption due to hospital noises: a prospective evaluation. Ann Intern Med 2012;157:170-9. 10.7326/0003-4819-156-12-201208070-00472 22868834

9 Hagerman I, Rasmanis G, Blomkvist V, Ulrich R, Eriksen CA, Theorell T. Influence of intensive coronary care acoustics on the quality of care and physiological state of patients. Int J Cardiol 2005;98:267-70. 10.1016/j.jijcard.2003.11.006 15686777

10 King's College London. Hospital project on noise, sound and sleep. 2017. www.kcl.ac.uk Cultural/-/Projects/Hospital-Project-on-Noise-Sound-and-Sleep.aspx.

11 Mackrill J, Jennings P, Cain R. Exploring positive hospital ward soundscape interventions Appl Ergon 2014;45:1454-60. 10.1016/j.apergo.2014.04.005 24768090

12 Mackenzie DJ, Galbrun L. Noise levels and noise sources in acute care hospital wards. Build Serv Eng Res Tech 2007;28:117-3110.1177/0143624406074468.

13 Mackrill J, Cain R, Jennings P. Experiencing the hospital ward soundscape: towards a model. J Environ Psychol 2013;36:1-810.1016/j.jenvp.2013.06.004.

14 Wynne J. ITU: the din of recovery. In: Minevich P, Waterman E, eds. The Art of Immersive Soundscapes. University of Regina Press, 2012: 128-41.

15 Murphy G, Bernardo A, Dalton J. Quiet at night: implementing a nightingale principle. Am J Nurs 2013;113:43-51. 10.1097/01.NAJ.0000438871.60154.a8 24284585

16 Farrehi PM, Nallamothu BK, Navvab M. Reducing hospital noise with sound acoustic panels and diffusion: a controlled study. BMJ Qual Saf 2016;25:644-6. 10.1136/bmjqs-2015-004205 26208539

17 Wilson C. Improving the patient's experience with a multimodal quiet-at-night initiative. $J$ Nurs Care Qual 2017;32(2):134-40. 10.1097/NCQ.0000000000000219 28225532

18 Richardson A, Thompson A, Coghill E, Chambers I, Turnock C. Development and Improving the patient's experience with a multimodal quiet-at-night initiative implementation of a noise reduction intervention programme: a pre- and postaudit of three hospital wards. J Clin Nurs 2009;18:3316-24. 10.1111/j.1365-2702.2009.02897.x 19732242

19 Gallacher S, Enki D, Stevens S, Bennett MJ. An experimental model to measure the ability of headphones with active noise control to reduce patient's exposure to noise in an intensive care unit. Intensive Care Med Exp 2017;5:47. 10.1186/s40635-017-0162-1 29043462

20 Farokhnezhad Afshar P, Bahramnezhad F, Asgari P, Shiri M. Effect of white noise on sleep in patients admitted to a coronary care. J Caring Sci 2016;5:103-9. 10.15171/jcs.2016.011 27354974

Published by the BMJ Publishing Group Limited. For permission to use (where not already granted under a licence) please go to http://group.bmj.com/group/rights-licensing/ permissions 